\title{
SURRALLÉS Alexandre, Au cœur du sens : perception, affectivité, action chez les Candoshi
}

\section{Emmanuel Désveaux}

\section{OpenEdition}

Journals

Édition électronique

URL : https://journals.openedition.org/jsa/1791

DOI : 10.4000/jsa. 1791

ISSN : $1957-7842$

Éditeur

Société des américanistes

Édition imprimée

Date de publication : 5 juin 2004

Pagination : 200-202

ISSN : 0037-9174

\section{Référence électronique}

Emmanuel Désveaux, « sURRALLÉs Alexandre, Au cœur du sens : perception, affectivité, action chez les Candoshi », Journal de la Société des américanistes [En ligne], 90-2 | 2004, mis en ligne le 17 novembre 2005, consulté le 02 septembre 2022. URL : http://journals.openedition.org/jsa/1791 ; DOl : https:// doi.org/10.4000/jsa. 1791

Ce document a été généré automatiquement le 2 septembre 2022

Tous droits réservés 


\title{
SURRALLÉs Alexandre, Au cœur du sens : perception, affectivité, action chez les Candoshi
}

\author{
Emmanuel Désveaux
}

\section{RÉFÉRENCE}

SURRALlÉS Alexandre, Au coeur du sens : perception, affectivité, action chez les Candoshi, préface de Philippe Descola, CNRS Éditions/Éditions de la Maison des sciences de l'homme, coll. « Chemins de l'ethnologie », Paris, 2003, 278 p., 6 p. de pl. coul., bibl., ill., tabl., fig., cartes

Le territoire des Candoshi embrasse le bassin inférieur du fleuve Pastaza, au nord de l'Amazonie péruvienne. Il s'agit d'un espace sylvestre équatorial où l'élément aquatique prédomine: lacs, marais et cours d'eau abondent. La navigation demeure dans ces conditions, même de nos jours, le principal moyen de pénétrer et de parcourir cet espace. Du coup, il est resté relativement isolé : les Candoshi présentent encore aujourd'hui bien des traits d'une société traditionnelle. Ajoutons qu'ils avaient été assez peu étudiés, sinon dans l'optique d'établir, sur les bases de comparaisons portant sur leur langue et de quelques traits d'organisation sociale, leur relation avec les fameux groupes jivaros dont les territoires s'étendent beaucoup plus au nord, de l'autre coté de la frontière avec l'Équateur. Dès lors, quelle perspective de terrain plus exaltante pouvait se dessiner pour un jeune ethnologue qui, de surcroît, travaillait sous la direction d'un des meilleurs spécialistes mondiaux des études jivaros? Cela étant, ce terrain se promettait d'être difficile. Nul doute qu'il le fut, ainsi que le laisse entendre avec beaucoup de pudeur et une pointe d'humour, peut-être involontaire, Alexandre Surrallés au détour d'une note de bas de page dans laquelle il dresse la liste des plaies du monde candoshi. Celles-ci vont du voisinage avec une guérilla prétendument révolutionnaire - et donc de la présence 
des forces armées censées la contrer-, aux multiples épidémies, en passant par une politisation, sous la houlette des missionnaires de diverses tendances, des élites locales.

Les Candoshi ont un mode d'existence sociale dont les traits saillants sont, d'une part, la figure de "grands hommes", d'autre part, une propension à la guerre de tous (ou presque tous) contre tous. L'interdépendance est forte entre les deux : il faut des grands hommes pour avoir la force morale de tuer et le meurtre rehausse, par effet de retour, le statut de leur auteur. De ce point de vue, l'ontologie a prise directe sur le sociologique, une pratique et une idéologie généralisée de la prédation occupant le centre du dispositif. L'importance de la prédation apparaît d'autant plus que l'économie des Candoshi - du moins son versant masculin qui sert incontestablement de référent repose sur la chasse. Il en résulte que le niveau de ce que nous appelons l'organisation sociale tend à se dissoudre. Le cas des Candoshi n'est pas exceptionnel en Amérique. À bien des égards, la lecture du texte de Surrallés a été très évocatrice pour moi, familier des chasseurs algonquins décrits naguère par Hallowell en termes d'atomisation sociale extrême. Le cas n'est pas exceptionnel, la situation semble assez similaire par exemple chez les Jivaros, bien qu'ici le lien social soit encore plus distendu, du moins dans l'espace. Surrallés, très opportunément, s'est gardé de se focaliser d'emblée sur cette question du sociologique. À l'opposé, il trouve dans le thème de l'individu, et plus spécifiquement encore du corps, l'entrée en matière de son propos. Dans ses premiers chapitres, il cherche ainsi à définir un rapport au monde qui passe avant tout par cet individu et ses états psychiques, lesquels sont immédiatement reliés à sa corporalité. "On voit avec le cœur» disent les Candoshi, et Surrallés de mettre en avant la perception - perception ancrée dans l'organe à la fois le plus interne et le plus fonctionnel que nous possédions - comme primat de l'intellection. Il en tire une phénoménologie de ce qu'il appelle la présence-prégnance de l'individu, qui « pèse » ipso facto sur le monde en vertu de son existence laquelle ne se justifie par rien d'autre in fine que le fait de vivre, alors même qu'il est exposé en permanence aux attaques potentielles des autres, de tous les autres, ses ennemis. Mais cette sorte d'égotisme prédatif qui caractérise l'éthos candoshi a pour pendant une théorie animiste qui prête aux entités autres, qu'elles soient naturelles ou sociales - ici la distinction ne joue guère -, une même capacité potentielle d'être au monde avant tout en tant que désir prédatif. La dentition, dont chacun est doté, certes de façon plus ou moins prononcée, signale la généralisation de cette propension à dévorer autrui comme condition d'existence du soi. On retrouve là une nouvelle illustration du perspectivisme amérindien dont les travaux de Viveiros de Castro ont récemment souligné l'importance.

Qu'il expose le savoir objectif que les Candoshi possèdent sur les corps, tous les corps à commencer par celui de l'abeille biibi; qu'il relate des situations relevant du quotidien, tel le retour de la chasse - où la prédation se joue sur un mode mineur-, tel encore le lent écoulement pour toute une maisonnée à moitié assoupie des heures les plus chaudes de la maison qu'il s'attache au contraire à évoquer d'autres situations plus exceptionnelles, tel l'accueil de l'étranger, moment d'extrême tension, car potentiellement dangereux; qu'il nous restitue les circonstances - toujours liées à la guerre - et les diverses étapes de la quête d'aruntam, cet esprit tutélaire lié à une sorte d'ancestralité patrilinéaire ; ou enfin qu'il nous rapporte la préparation et la conduite des expéditions guerrières elles-mêmes, et ce avec une très grande richesse de détails au point de presque sombrer dans le gore, Surrallés excelle à mettre l'individu et sa corporalité au centre de son ethnographie, appuyant ainsi par le jeu de l'écriture, son 
projet théorique d'une phénoménologie exotique. Cette qualité quasi herméneutique du texte tient également à l'exploration récurrente de champs lexicaux.

Cela étant, au fur et à mesure qu'il développe son argument, Surrallés est rattrapé par les questions d'organisation sociale. Il rejoint alors les idées qui prévalent aujourd'hui chez la plupart de nos collègues amazonistes, en particulier au sujet de l'échange matrimonial comme fondement de l'ordre social. Il est clair toutefois qu'ici la doxa sociologique lévistraussienne entre en contradiction avec le modèle de l'individualisme prédatif qui semble caractériser les Candoshi, du moins si l'on s'en tient aux - remarquables descriptions et analyses de Surrallés lui-même. Globalement, on ne saurait dire que cette contradiction le préoccupe outre mesure. Cela étant, on perçoit par endroits comme un léger scepticisme face au dogme dominant, par exemple au détour d'une discussion sur les nomenclatures de parenté. Nous soulignerons de notre côté que son ajustement à ce dogme l'empêche peut-être de commenter certains phénomènes qu'il rapporte pourtant, comme le caractère très souvent incestueux des premières amours ou l'impossibilité pour le fratricide de réintégrer dans le tissu social, au contraire de toutes les autres catégories de meurtriers ou, si l'on préfère, de guerriers. Entendons-nous bien, ce n'est pas la «sanction» qui suscite la perplexité, mais bien l'existence même de ce type de meurtrier. Comme si la dynamique de la violence et de l'appropriation-prédation (car, en définitive, l'appropriation, la prise de contrôle de la victime est inhérente à toute prédation; on ne dévore que ce dont on dispose) débordait du cadre mécaniciste de la relation équilibrée entre le local et l'extra-local. Peut-être eût-il été pertinent alors de regarder du côté de Clastres, lui qui avait dit, en dépit de ses outrances, des choses fortes sur le sujet...

$5 \mathrm{Au}$ fond, ces divers points sont mineurs, surtout par rapport au projet de Surrallés. Dans son introduction, celui-ci affiche en effet une ambition avant tout monographique. Au cœur du sens témoigne magnifiquement de sa réussite: avec cet ouvrage, nous tenons entre les mains une de ces très belles monographies que notre discipline a su, à intervalle régulier, produire. À quelques statistiques, graphiques et tableaux près, ce texte rigoureux et captivant à la fois, où descriptions et analyses se répondent harmonieusement, nous fait penser à ces grands textes d'ethnologie américaniste qui honorent le catalogue de la collection "Terre humaine». Nous n'avons qu'un regret: qu'il ne soit pas venu l'enrichir, se donnant ainsi la chance de rencontrer un public élargi.

\section{AUTEURS}

\section{EMMANUEL DÉSVEAUX}

EHESS/Musée du quai Branly, Paris 